\title{
Survey on Fault-Tolerant Diagnosis and Control Systems Applied to Multi-motor Electric Vehicles
}

\author{
Alexandre Silveira $^{1}$, Rui Esteves Araújo ${ }^{2}$, and Ricardo de Castro ${ }^{2}$ \\ ${ }^{1}$ Instituto Superior de Engenharia do Porto \\ ${ }^{2}$ Faculdade de Engenharia, Universidade do Porto, Portugal \\ asi@isep.ipp.pt, \{raraujo,de.castro\}@fe.up.pt
}

\begin{abstract}
In the last years we have witnessed a growing interest, by the academic community and the automotive industry, in the multi-motor electric vehicles. The electrical nature of the propulsion is going to stress even more an increasing insertion of electronic devices in the vehicles. Furthermore, carmakers are performing research and already presented some vehicles based on the concept of X-By-Wire. Consequently, the growing complexity of the actuators and their control, as well as the need of increasing the safety and reliability of the vehicles obliges to the study and development of intelligent computational systems dedicated to the detection and diagnosis of failures in the electric propulsion. Hence, it is fundamental to start advanced studies leading to the development of innovative solutions that embed fault-tolerant electric propulsion in the electric vehicles. Accordingly, the main objective of this work consists on the bibliographic revision and study of fault-tolerant diagnosis and control systems dedicated to multi-motor electric vehicles.
\end{abstract}

Keywords: Fault detection and diagnosis, Fault tolerant control systems, multimotor electric vehicles.

\section{Introduction}

As electric vehicles are systems with propellers based in electromechanical drives, we can classify them as critical systems, where the use of fault-tolerant control techniques becomes essential. As referred in [1] and [2], the interest of introducing fault detection, tolerance and redundancy in a system is to increase its safety and reliability. A system is considered to be safe if it is able to prevent any danger to humans, equipments and environment; and is reliable if it is capable of perform correctly the required functions, over a certain period of time under a given set of conditions. These characteristics are of great importance in safety related processes and systems like aircrafts, trains, automobiles and power plants [2].

So, in order to improve system's reliability and operational safety, reducing the possibility of those failures or trying to predict its happening before occurrence it's necessary. One way to do this is to employ Fault Detection and Diagnosis systems (FDD). The FDD consists of making a binary decision when something wrong happens, and to determine the location and the nature of the fault. These methods are 
based on the concept of redundancy, which can be obtained with hardware or using analytical redundancy. In the former, backup sensors and/or actuators are used in such a manner that the system is able to automatically replace the faulty ones when a given fault is identified. This strategy is not always possible due to physical or economical constrains. An alternative way to turn the system more reliable is to use the concept of analytical redundancy that uses a mathematical model and estimation techniques. With this kind of concept, the system is able to shut down itself or to employ adequate procedures to tolerate the faults and maintain the system operational [3].

This paper is structured as follows: In section 2 it is shown the contribution to sustainability of this paper. In section 3 it is made an overview on the Fault Detection and Diagnosis approaches for implementation in electric vehicles (EVs) and in section 4 there are presented some conclusions about this survey.

\section{Contribution to Sustainability}

The interest in the electric vehicles rose recently due both to environmental questions and to energetic dependence of the contemporary society [4]. Moreover, in the passenger car industry, the majority of the constructors are currently developing considerable efforts to introduce the first generation of pure electric vehicles, like the Nissan Leaf, the Mitsubishi iMIEV or the Fluence Z.E. of Renault. In fact, some are already available in some European markets. Accordingly, it is necessary to study and implement in these new vehicles fault-tolerant control systems which enable them to be more reliable and safe, enhancing its sustainability.

\section{Overview on Fault Detection and Diagnosis for EVs}

The fault detection and diagnosis techniques are well studied in the specialty literature [1], [5] and [6], and are more and more applied in industrial processes and products. Therefore, the actual state of the technique motivates the next step, which consists in the following: after the fault detection the controller must be capable of guarantee the functioning of the vehicle in safety conditions. In other words, it is necessary to study and develop fault-tolerant control architectures dedicated for the future electric vehicles.

Lately, as we are getting more and more concerned about safety, reliability and sustainability, there was a rise in the research of fault detection and diagnosis systems, that led to the development of many FDD techniques [1], [7]. Although these technical and scientific progresses, the conception of fault-tolerant controllers oriented to electric vehicles is simultaneously a complex and fascinating project. The reasons for this are inherent to the difficulties of controlling in an efficient, effective and secure way the several propellants in a multi-motor electric vehicle.

Detailed analysis of faults processes has indicated the need to act quickly following a device failure to prevent propagation of faults that may lead to catastrophic failure of the propulsion system. To minimize the effect of fault, it is essential to accurately identify the failed devices and its mode of failure. Historically, in what concerns practical applications, a great amount of research on fault-tolerant control systems 
was derived from the aerospace industry [1]. Nowadays is recognized some maturity to the theoretical concepts of the fault-tolerant controllers, whose one of the pioneers was Ron Patton [8], where he makes a bibliographical revision encompassing the principal fault-tolerant systems control areas.

The works of Mutoh and Nakano [9], demonstrate by simulations that for electric multi-motor vehicles, faults in one of the propulsion systems lead to the loss of stability of the vehicle. Thus, their work motivates for the necessity of study and evaluation of different configurations regarding the number of motors and their localization in the vehicle. The possibility of the inclusion of the motor in the wheel will contribute to the conceptualization, validation and implementation of new innovative active torque distribution methodologies for the future vehicles, potentiating improvements in their handling, safety and stability. However, this innovative solutions lead to new challenges due to the fact that the electric motors are feed by electronic power converters that have a bigger fault probability. In the case of Electric Hybrid Vehicles, if the motor is not monitored, motor faults might lead to severe damages or even accidents [10].

Considering the electric vehicles as critical systems, with propellers based in electromechanical drives, the use of fault-tolerant control techniques is essential. On the other hand, the adoption of the "X-by-wire" technology, where " $\mathrm{X}$ " represents the several subsystems to control in the vehicle, as "Steer-by-wire", "Throttle-by-wire" or "Brake-by-wire", motivate to the study of fault-tolerant controllers [11], [12].

In the literature we can find several solutions in the thematic of the fault-tolerant electromechanical actuators, with special emphasis in the electronic converters and motors [13]. The work of Delgado [14] presents a review of fault-tolerant systems of electronic speed drives to DC motors and induction motors and some hardware configurations. Also, Murphrey et al. [15] presents a fault diagnosis Neural Network system that detects and locates multiple classes of faults in electric drives, using a machine learning technology.

Regarding traction applications, Akin [10] presents a fault diagnosis system to electric or hybrid vehicle's motors, based on the Fast-Fourier Transform (FFT) method. In four wheel steer/four wheel drive systems (4WS4WD) we must stress the work of Yang [16] that proposes a fault-tolerant hybrid approach to maintain the vehicle in a functional state even in the presence of a failure. Thus, there is an increasing demand for dynamic systems to operate autonomously in the presence of faults and failures in sensors, actuators and components. So, fault detection and diagnosis are essential components in an autonomous fault tolerant control system [17]. Therefore it is necessary to design control systems capable of tolerate possible faults in those systems, in order to improve reliability and availability [18].

A Fault-Tolerant Control Systems (FTCS) is a control system capable of accommodating system component faults (actuators, sensors) and able to guarantee stability and an acceptable degree of performance. FTCS can also prevent that faults in a subsystem may develop into failures at the system level [18]. Fault-Tolerant Control Systems (FTCS) can be classified into two types: passive (PFTCS) and active (AFTCS). Contrasting with the former, the latter react with system component failures actively and implement reconfiguring actions to maintain the system stable and with acceptable performance [1]. AFTCS are usually constituted of four subsystems: a reconfigurable controller, a FDD scheme, a controller reconfiguration mechanism and a command/reference governor [1], as illustrated in Fig. 1. 


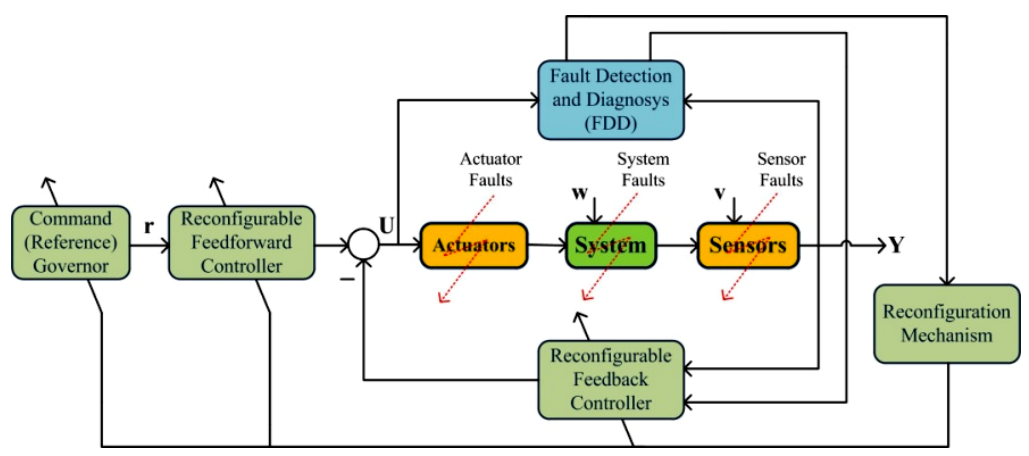

Fig. 1. General structure of AFTCS [1]

The FDD block represented above is capable of detecting the presence of faults in the system that it monitors, is able to determine their locations and can estimate their severities. In other words, it is capable of doing three tasks [17]:

- Fault detection: to decide if everything is working like expected or something has gone wrong;

- Fault isolation: to determine the location (component, sensor, actuator) of the fault;

- Fault identification: to estimate the severity, type or nature of the fault.

The reconfigurable controller should be designed automatically to compensate the fault-induced changes in the system in order to maintain their stability and closedloop system performance [1].

In the recent years, many research and work have been done in the area of FDD. One example is the paper of Zhang and Jiang [1] were they presented a classification of several FDD methods. As explained by them, FDD approaches are usually classified into two categories: (1) model-based and (2) data-based (model-free) schemes. Moreover, these two schemes can also be classified into qualitative and quantitative approaches. Relatively to this classification, Muenchhof [7] and Liu [19] refer that classical model-free methods rely on hardware redundancy, resulting on extra hardware, cost and size but on the other hand can result in reduction of unexpected downtime of the system. Contrarily, model-based methods rely on analytical redundancy, where consistency between the expected behavior and measurements of the process is checked based on analytical models [19]. As reported in [18], model-based methods are best suited for processes which input and output signals can be measured. However, if we can only measure the outputs of a process, signal-based methods should be applied.

\subsection{Model-Based Methods}

Model-based fault detection methods are well studied and reported in the literature [2], [7], [19], [20], [21] and [22]. According to [18], model-based methods are widely used and are usually performed in two steps: residual generation and residual evaluation. In this kind of method, the difference between the measurement and the 
expected behavior is called residual. As mentioned by Isermann [2], this consists of the detection of faults of processes, actuators and sensors by using dependences, expressed by mathematical process models, between different measurable signals. The basic structure of model-based fault detection is illustrated in Fig. 2.

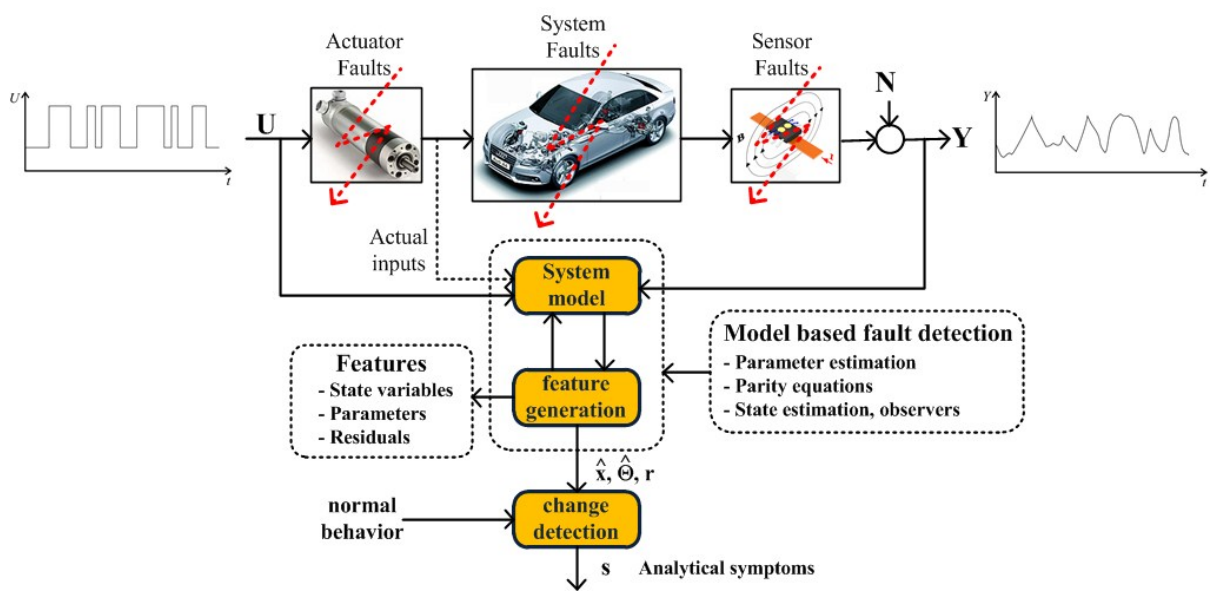

Fig. 2. Basic structure of model-based and signal based FD [2]

Based on the measured input signals $\mathbf{U}$ and output signals $\mathbf{Y}$, the detection methods generate the residuals $\mathbf{r}$, parameter estimates $\hat{\Theta}$ or state estimates $\hat{x}$, which are called features. Comparing them with normal features, changes are detected, leading to analytical symptoms $\mathbf{s}$ [2]. This analytical symptom generation is quantifiable and analytical information about the process, and is a result from the data processing of the measured signals [18]. We can divide model-based methods in four main classes: state estimation approaches, parity space approaches, parameter estimation approaches and simultaneous state/parameter estimation approaches. In first class, the system outputs are estimated from measurements using: Luenberger observer, linear or nonlinear observers, sliding-mode observers and high-gain nonlinear observers, for deterministic cases. In the case of stochastic ones, outputs are estimated using: Kalman filter (linear, extended and unscented) or receding horizon estimators [17] and [18].

In the parity space approaches, residual are computed as difference between measured outputs and estimated outputs and their associated derivatives. In the parameter estimation approach, residuals are computed as the parameter estimation error, by continuously estimating the parameters of a process model [18]. Parity space methods are based on simple algebraic projections and geometry and are more sensitive to measurement noise and process noise as compared to observer-based methods [17]. Still, these methods are mainly suitable for detection and isolation of additive faults. They are simpler and easy to implement compared to observer based techniques. Parameter estimation approach is based on the assumption that the faults are reflected in the physical parameters of the system. In this approach the system parameters are estimated online with parameter estimation techniques [17]. 
In this model-based approach there are also some works related to the problematic of safety in X-by-Wire systems such as drive-by-wire, steer-by-wire, throttle-by-wire or brake-by-wire [11], [12], [21] [23], and [24].

Most of fault-tolerant control systems require hardware redundancy to make them more reliable [11], [12], [24] and [25]. This means that one or more modules are connected, usually in parallel. Such redundant schemes can be implemented for hardware, software, information processing and mechanical and electric components like sensors, actuators, microcomputers or power supplies [12]. Regarding these redundant structures, there are two basic approaches for fault tolerance: (1) static redundancy and (2) dynamic redundancy. Static Redundancy uses multiple redundant modules with majority voting (all modules are active). Dynamic redundancy requires fewer modules at the cost of more information processing. However, without mechanical redundancy, the reliability of the system needs to be improved by implementing fault-tolerant control techniques [21]. In fact, with the profit margin already low, mechanical redundancy will not be acceptable to the automobile industries.

Employing analytical redundancy techniques instead of hardware redundancy it will be possible to reduce costs, volume and weight to a point where the automakers feel comfortable, without compromising safety and reliability required by consumers [26]. According to [20] the concept analytical redundancy stands generally for an analytical reconstruction of quantities or parts of the system or process under monitoring. For Anwar [24], the concept of analytical redundancy has been investigated to replace hardware redundancy, in order to reduce overall cost and at the same time to improve reliability.

The above discussed techniques of model based approaches to fault diagnosis are powerful if an accurate system model is available. The diagnostic performance may be limited when it is not possible to obtain accurate and robust models.

\subsection{Data-Based Methods}

An alternative approach to the model-based residual generation is the data-based approach, also called model-free approach. This learning-based method learns the plant model from an historical input and output data of the system, i.e., it detects faults by analyzing specific properties of measured signals. According to [17] databased methods are based on signal processing techniques, obtained using either of the following two types: (1) Time domain limit checking and/or trend analysis and (2) Frequency or mixed time-frequency domain analysis. In the former, the statistics of the measurable states and outputs of the system are compared with nominal operating limits. In the latter, it is made an analysis of the time series of system states and outputs measured by system sensors [17]. In the time domain, the most common technique is the Qualitative Trend Analysis (QTA), while in frequency domain the most widely used algorithms are the Discrete Fourier Transform (DFT), and the Discrete Wavelet Transform (DWT) [17].

For [17], the major drawback of these signal processing techniques is that they do not consider the dynamic interrelationship between the different measured signals of the system. Thus, these techniques are more appealing for situations where highfidelity mathematical model of the monitored system does not exist or is very difficult 
to obtain. According to the same author, artificial neural networks, fuzzy logic and neuro-fuzzy systems are the most widely used approaches. Using these techniques we can work on the quantitative and qualitative information of the monitored system. Qualitative information is expressed in the form of Boolean or fuzzy if-then rules. These have a drawback that is the problem of deriving Boolean or fuzzy if-then rules in many engineering applications. In fact, this requires extensive expert knowledge of the system [17]. On the other hand, Neural Networks (NN) are ideal mathematical tools for situations like this, where the knowledge that describes the behavior of the system is stored in large quantitative datasets [17]. Also, as mentioned by Patton [27], a well trained NN has the capacity of making intelligent decisions even in the presence of noise, system disturbances and corrupted data.

\section{Conclusions}

Fault Detection and Diagnosis are systems of great importance for modern electric vehicles. This is even more critical for multi-motor electric vehicles, since their stability is deteriorated in the presence of fault in one of the thrusters. As their thrusters are constituted by the electronic power converters and the motors, it is necessary to study the faults in these systems and to implement FTCS to deal with them, maintaining safety for their users and ensure sustainable operation.

In summary, even though there are some pioneering works, there are not known published ones describing the application of fault-tolerant control techniques, especially with a holistic view on the multi-motor electric vehicles.

\section{References}

1. Zhang, Y., Jiang, J.: Bibliographical review on reconfigurable fault tolerant control systems. Annual Reviews in Control, 229-252 (2008)

2. Isermann, R.: Model-based fault-detection and diagnosis - status and applications. Annual Reviews in Control, 71-85 (2004)

3. Li, X.: Fault Detection Filter Design for Linear Systems, PhD Dissertation (August 2009)

4. Chan, C., Chen, K.: Electric, Hybrid, and Fuel-Cell Vehicles: Architectures and Modeling. IEEE Transactions on Vehicular Technology 59, 589-598 (2010)

5. Blanke, M., Kinnaert, M., Lunze, J., Staroswiecki, M.: Diagnosis and Fault Tolerant Control, 2nd edn. Springer, Heidelberg (2006)

6. Patton, R., et al.: Issues of Fault Diagnosis for Dynamic Systems. Springer, Heidelberg (2000)

7. Muenchhof, M., Beck, M., Isermann, R.: Fault-tolerant actuators and drives - Structures, fault detection principles and applications. Annual Reviews in Control (2009)

8. Patton, R.: Fault-tolerant control: the 1997 situation. In: IFAC Symposium on Fault Detection, Supervision and Safety for Technical Processes (SAFEPROCESS1997), Hull, UK, vol. 2, pp. 1033-1055 (1997)

9. Mutoh, N., Nakano, Y.: Dynamic Characteristic Analysis of the Front- and Rear-Wheel Independent-Drive-Type Electrical Vehicle (FRID EV) When the Drive System Failed during Running under Various Road Conditions. In: IEEE Vehicle Power and Propulsion Conference VPPC 2009 (2009) 
10. Akin, B., Ozturk, S.B., Toliyat, H.A., Rayner, M.: DSP-Based Sensorless Electric Motor Fault-Diagnosis Tools for Electric and Hybrid Electric Vehicle Powertrain Applications. IEEE Transactions on Vehicular Technology 58(6) (2009)

11. Naidu, M., Gopalakrishnan, S., Nehl, T.: Fault Tolerant Permanent Magnet Motor Drive Topologies for Automotive X-By-Wire Systems (2009)

12. Iserman, R., Schwarz, R., Stölzl, S.: Fault tolerant drive-by-wire systems. IEEE Control Systems Magazine, 64-81 (2002)

13. El Hachemi Benbouzid, M., Diallo, D., Zeraoulia, M.: Advanced Fault-Tolerance Control of Induction-Motor Drives for EV/HEV Traction Applications: From Conventional to Modern and Intelligent Control Techniques. IEEE Transactions on Vehicular Technology 56(2) (2007)

14. Delgado, D., et al.: Fault-Tolerance Control in Varaiable Speed Drives: A Survey. IET Electric Power Applications 2, 121-134 (2008)

15. Murphey, Y.L., Masrur, M.A., Chen, Z., Zhang, B.: Model-based fault diagnosis in electric drives using machine learning. IEEE/ASME Transactions on Mechatronics 11(3), 290-303 (2006)

16. Yang, H., Cocquempot, V., Jiang, B.: Hybrid Fault Tolerant Tracking Control Design for Electrical Vehicles. In: IEEE 16th Mediterranean Conference on Control and Automation (2008)

17. Sobhani-Tehrani, E., Khorasani, K.: Fault Diagnosis of Nonlinear Systems using Hybrid Approach. Springer, Heidelberg (2009)

18. Mahmoud, M.M., Jiang, J., Zhang, Y.: Active Fault Tolerant Control Systems: Stochastic Analysis and Synthesis. Springer, Heidelberg (2003)

19. Liu, L., Logan, K.P., Cartes, D.A., Srivastava, S.K.: Fault Detection, Diagnostics, and Prognostics: Software Agent Solutioins. IEEE Transactions on Vehicular Technology 56(4), 1613-1622 (2007)

20. Ding, S.X.: Model-based Fault Diagnosis Techniques: Design Schemes, Algorithms, and Tools. Springer, Heidelberg (2008)

21. Im, J.S., Ozaki, F., Yue, T.-K., Kawaji, S.: Model-based fault detection and isolation in steer-by-wire vehicle using sliding mode observer. Mechanical Science and Technology, 1991-1999 (2009)

22. Dumont, P.E.: Fault Detection of Actuator Faults for Electric Vehicle. In: 16th IEEE International Conference on Control Applications, Singapore, pp. 1067-1072 (2007)

23. Laboratory, Army Research. Using a Steering Shaping Function to Improve Human Performance in By-Wire Vehicles. Document (August 2009),

http://www. defensetechbriefs.com/component/content/article/ 5554

24. Anwar, S., Chen, L.: An Analytical Redundancy-Based Faut Detection and Isolation Algorithm for a Road-Wheel Control Subsystem in a Steer-By-Wire System. IEEE Transactions on Vehicular Technology 56(5), 2859-2869 (2007)

25. Hwang, I., Kim, S., Kim, Y., Chze, E.S.: A Survey of Fault Detection, Isolation, and Reconfiguration Methods. IEEE Transactions on Control Systems Technology 18(3), 636652 (2010)

26. Hasan, M.S., Anwar, S.: Sliding Mode Observer Based Predictive Fault Diagnosis of a Steer-By-Wire System. In: 17th World Congress of the International Federation of Automatic Control, Seoul, Korea, pp. 8534-8539 (2008)

27. Patton, R.J., Lopez-Toribio, C.J.: Artificial intelligence approaches to fault diagnosis. IEEE Colloquium on Update on Developments in Intelligent Control (Ref. No. 1998/513), 311-312 (October 23,1998) 